\title{
Physikalische Therapie: Neurotransmitter
}

\author{
Jean-Michel Jeannin
}

Basel, Schweiz

\section{Einleitung}

Mittel der Physikalischen Therapie können neben den körperlichen Zuständen, zu deren Behandlung sie eingesetzt werden, auch die Psyche der behandelten Patienten günstig beeinflussen: Besserung von Depressionen, Angstzuständen und Schmerzen. Auch kognitive Funktionen können mittels Physikalischer Therapie beeinflusst werden. Solche Wirkungen erscheinen zwar evident, beruhen aber auch auf konkreten biochemischen und neuroanatomischen Vorgängen. Dazu gehört die Beeinflussung von Neurotransmitter-Konzentrationen und von neuroplastischen Vorgängen. In den folgenden Beiträgen der Reihe werden einige der Mechanismen beschrieben, die sich sozusagen im Hintergrund einer Physikalischen Therapie abspielen (Abb. 1, 2).

\section{Psychiatrische Störungen}

Es herrscht ein gewisser Konsens darüber, dass körperliche Anstrengung inklusive definiertem Training eine günstige Wirkung auf eine Depression ausübt. Darüber, welche zentralnervösen Mechanismen auf der Ebene von

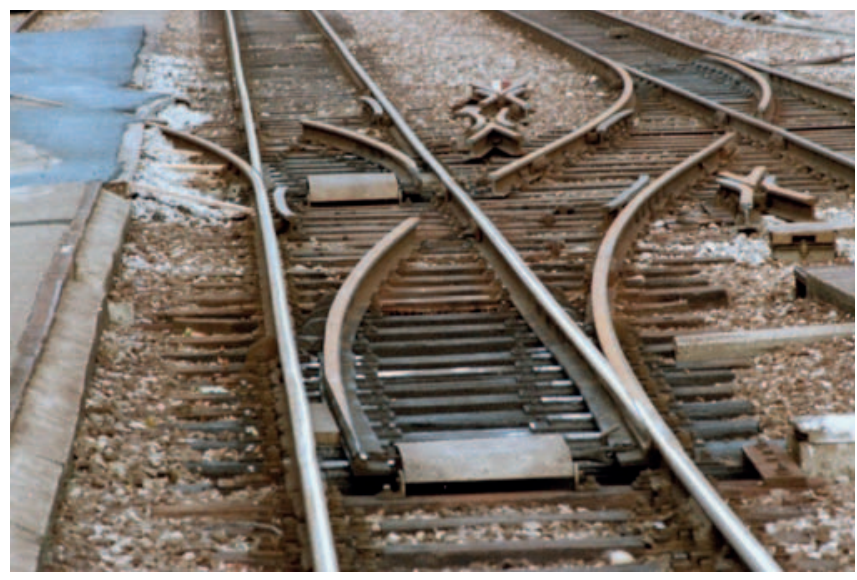

Abb. 1. Unterbrochene Wege (Gleisbau in Oberwil BL, 1984).

\section{KARGER}

(c) 2017 S. Karger GmbH, Freiburg

Fax +497614520714
Neurotransmittern involviert sind, gibt es eine gewisse Evidenz. Definitive Beweise sind aus methodologischen Gründen schwierig zu erbringen. Häufig erwähnt werden die Endorphin-Hypothese und die Monoamin-Hypothese [1] Gemäss diesen Hypothesen sind $\beta$-Endorphin bzw. Dopamin, Noradrenalin und Serotonin involviert. Dies lässt sich in Tierversuchen gut nachweisen; der Zusammenhang zwischen Änderungen des Blutspiegels dieser Substanzen und den Änderungen zentralnervöser Funktionen beim Menschen lässt sich jedoch nur schwer untersuchen.

Shevchuk [2] präsentiert seine Hypothese, wonach zwei konvergierende Faktoren eine Depression verursachen können: Ein Mangel an Stressoren sowie eine genetisch bedingte Veranlagung der Betroffenen, auf einen Mangel an Stressoren stärker als andere zu reagieren. Solche Stressoren sind z.B. häufige körperliche Tätigkeiten oder häufige Änderungen der Körpertemperatur, z.B. beim Schwimmen in kaltem Wasser oder Jagen in heissem Klima. Die Haut enthält 3- bis 10-mal so viel Kälte- wie Wärmerezeptoren. Werden alle Kälterezeptoren gleichzeitig angeregt, z.B. beim Eintauchen in kaltes Wasser, wird eine besonders intensive Wirkung auf das Gehirn erzielt. Im Einzelnen aktiviert Kälte den Sympathikus und steigert den Gehalt von Noradrenalin im Blut sowie die

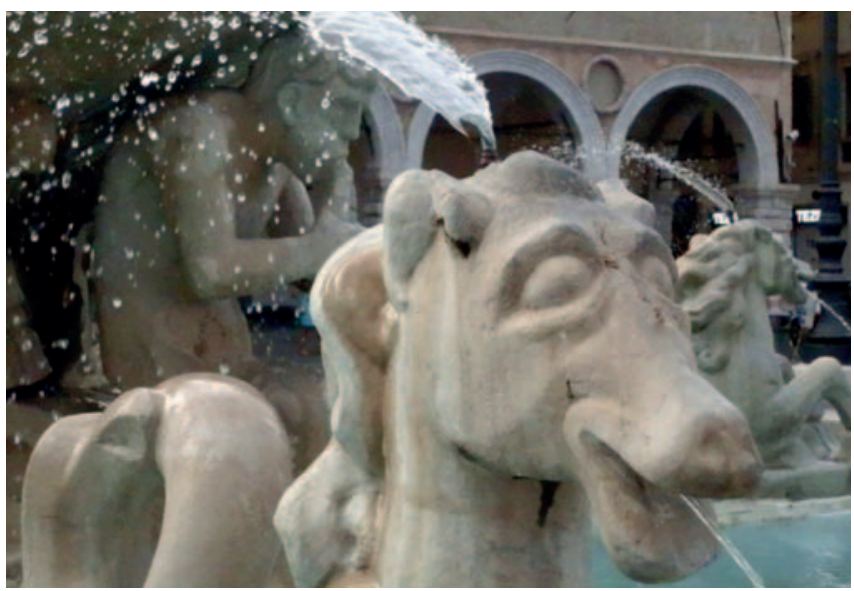

Abb. 2. Wasser und Gehirn (Brunnen in Pesaro, Italien, 2017).

Dipl. med. biol. Jean-Michel Jeannin

Holeestrasse 43, 4054 Basel, Schweiz

jm.jeannin@medwiss.ch 
Tab. 1. Glossar

\begin{tabular}{ll}
\hline Hippocampus & $\begin{array}{l}\text { Der Hippocampus ist ein entwicklungsgeschichtlich alter Teil des Grosshirns. Die Ähnlichkeit seiner Gestalt mit der eines Seepferd- } \\
\text { chens gab ihm den Namen. Der Hippocampus leitet sensorische Nervenimpulse aus der Peripherie (Afferenzen) an die Grosshirnrinde } \\
\text { weiter. Er ist in die Regulierung des Schlaf-Wach-Rhythmus sowie von Aufmerksamkeit und Konzentration involviert. Die Grosshirn- } \\
\text { hälften haben je einen Hippocampus. }\end{array}$ \\
\hline Gyrus dentatus & $\begin{array}{l}\text { Der Gyrus dentatus ist ein Teil des Hippocampus und bildet die Eingangspforte für die afferenten Impulse. Er nimmt eine Schlüssel- } \\
\text { rolle in der Umwandlung von Kurzzeitgedächtnisinhalten in Inhalte des Langzeitgedächtnisses ein. Er bildet lebenslang neue Nerven- } \\
\text { zellen. }\end{array}$ \\
\hline $\begin{array}{l}\text { Locus coeruleus } \\
\text { Region im Mittelhirn. Im Locus coeruleus befinden sich die meisten Noradrenalin-produzierenden Zellen des Gehirns. }\end{array}$ \\
\hline $\begin{array}{l}\text { p-Endorphin ist eines der drei körpereigenen Opioide. } \alpha \text {-, } \beta \text { - und } \gamma \text {-Endorphin regulieren die Schmerz- und Hungerempfindung und } \\
\text { soziale Interaktionen. }\end{array}$ \\
\hline $\begin{array}{l}\text { Noradrenalin ist ein Neurotransmitter und ein naher Verwandter des Adrenalins. Es wird in der Nebenniere sowie im Locus coeruleus } \\
\text { gebildet. Im Gehirn reguliert es den Aktivitätsgrad. }\end{array}$ \\
\hline $\begin{array}{l}\text { Funktionell entspricht 2-Phenylethylamin ( } \beta \text {-Phenylethylamin) endogenem Amphetamin. Die Konzentration von Phenylethylamin ist } \\
\text { im Vergleich zu der anderer Neurotransmitter sehr gering. Deshalb spricht man auch von einem Spurenamin. }\end{array}$
\end{tabular}

Freilassung von Noradrenalin im Locus coeruleus und im Hippocampus des Gehirns. Auch die Synthese von $\beta$-Endorphin wird gesteigert, was sich in Wohlbefinden äussert. Das gleiche Prinzip liegt der Elektrokonvulsionstherapie zugrunde: Das Gehirn wird kurzzeitig einem dosierten Stress ausgesetzt. Der Autor sieht in der Verabreichung von kalten Duschen eine mögliche Behandlung von Depressionen [2]. Für die Hypothese spricht eine gewisse evolutionsbiologische Evidenz. Für die Theorie als Ganzes ist die Richtigkeit aber noch nicht nachgewiesen.

Training und körperliche Aktivitäten haben günstige Wirkungen auf Depressionen, die mit der medikamentösen Therapie vergleichbar sind. Die Wirkungen werden zum Teil von $\beta$-Endorphin vermittelt, das bei hoher körperlicher Beanspruchung analgetisch wirkt [3].

Es herrscht Konsens, dass körperliche Aktivität, namentlich strukturiertes Training, antidepressiv wirksam ist. Der Neurotransmitter $\beta$-Phenylethylamin ist an der Regulierung der körperlichen Aktivität, der Stimmung und der Aufmerksamkeit beteiligt. Szabo et al. [4] konnten in einer Pilotstudie zeigen, dass aerobes Training die Bildung dieser Substanz stimuliert. Dazu haben sie die Ausscheidung von Phenylessigsäure im Urin gemessen, einem Metaboliten des $\beta$-Phenylethylamins. Die Bestimmungen wurden jeweils vor der Übung und am nächsten Tag nach der Übung durchgeführt. Die Übung bestand in 30 min Belastung auf dem Fahrradergometer bei 70\% Herzfrequenzreserve (ein Äquivalent für die maximale Leistung). Die mittlere Konzentration im 24-StundenUrin war nach der Übung um 77\% erhöht. Die Studie gibt einen Hinweis auf eine Verbindung von körperlicher Tätigkeit mit der Stimmung auf neurobiochemischer Ebene. Weitere Einzelheiten müssen noch untersucht werden.

In Tierversuchen konnte gezeigt werden, dass toxische Substanzen in geringer Dosis verabreicht sowie Hitze und Kälte in geringer Intensität angewendet die
Lebenserwartung der Versuchstiere verlängern können (sogenannte «Hormesis»). Chronische Erschöpfung ist mit einer Minderfunktion der Hypothalamus-Hypophysen-Nebennieren-Achse vergesellschaftet. Die Verarbeitung motorischer Impulse im Gehirn ist ebenfalls vermindert; gleichzeitig ist die Muskulatur der betroffenen Patienten schneller ermüdet. Kältestress aktiviert den Locus coeruleus, was gleichbedeutend mit einer Erhöhung des Noradrenalinspiegels ist. Kältestress aktiviert auch die Hypothalamus-Hypophysen-Nebennieren-Achse. In der Folge steigt der Blutspiegel des adrenocorticotropen Hormons (ACTH), was wiederum zu einer Erhöhung des Cortisol-Spiegels führt. Der Autor sieht die Kaltwassertherapie als Ersatz für die natürlichen Stimuli, die in der heutigen bewegungsarmen Lebensweise oft fehlen [5].

Körperliche Tätigkeit inklusive Training lösen psychotrope Wirkungen wie z.B. Glücksgefühle, Euphorie und Friedlichkeit aus, die den Wirkungen von $\Delta 9$-Tetrahydrocannabinol entsprechen. Diese Beobachtung veranlasste Tantimonaco et al. [6], eine Hypothese aufzustellen, wonach mindestens ein Teil der psychotropen Wirkungen von Bewegung und Training vom Endocannabinoiden vermittelt wird. Mit dem Endocannobinoid-System wurde ein weiteres Transmitter-System identifiziert. Endocannabinoide sind Ester, Amide und Ether der Arachidonsäure. Sie ahmen die Wirkung von $\Delta 9$-Tetrahydrocannabinol nach und binden an Typ-1- und Typ-2-Cannabinoid-Rezeptoren. Die am meisten untersuchte Substanz ist das NArachidonoylethanolamid (AEA). Der Blutspiegel von AEA nimmt durch ein Training mittlerer Intensität auf dem Laufband oder Fahrradergometer zu, nicht aber bei einem Training mit geringerer oder deutlich höherer Intensität. Die AEA-Spiegel konnten unter Bedingungen, wie sie in grossen Höhen gelten (Hypobarie, Hypoxie), noch gesteigert werden. 


\section{Serotonin (5-Hydroxitryptamin (5-HT))}

Nach gängiger Auffassung beruht die Depression auf einem Mangel an Serotonin, da die Gabe von SerotoninWiederaufnahme-Hemmern die Symptome lindern kann (Tab. 1). Die Wirkungsweise dieser Substanzen besteht in der Hemmung der Wiederaufnahme von Serotonin in die Synapsen, die es ausgeschieden haben. In der Folge bleibt mehr Serotonin im synaptischen Spalt für die Reizübertragung zur Verfügung. Das Modell wird z.B. von Andrews et al. [7] als reduktionistisch kritisiert. Die Autoren machen geltend, dass es verschiedene Formen von Depressionen gibt und nicht jede Form mit einem Serotoninmangel verbunden ist. Weiter weisen die Autoren darauf hin, dass derzeit 14 verschiedene Serotonin-Rezeptoren identifiziert wurden. Die Rezeptoren 5-HT1 und 5-HT5 vermitteln hemmende Wirkungen, die Rezeptoren 5-HT2, 5-HT3, 5-HT4, 5-HT6 und 5-HT7 stimulierende Wirkungen. Evolutionsbiologisch entwickelte sich Serotonin als Energieregulator. Depressive Zustände haben einen hohen Energiebedarf, weshalb Serotonin involviert ist. Serotonin kann die Blut-Hirn-Schranke nicht überwinden, was eine nichtinvasive Untersuchung der Serotoninspiegel im Gehirn unmöglich macht. In der Praxis wird auf Parameter wie das Verhältnis von Serotonin zu 5-Hydroxyindolessigsäure ausgewichen. Die Bestimmung dieses Parameters erlaubt eine Schätzung des Serotonin-Status im Gehirn.

\section{Reflexionen}

Bewegung und namentlich strukturiertes Training haben eine Reihe von Auswirkungen auf Neurotransmitter, darunter Dopamin, Histamin, Noradrenalin, $\beta$-Phenylethylamin und Serotonin. Analog wirkt sich z.B. eine Kaltwassertherapie auf bestimmte Transmitter aus.

Die sesshafte, bewegungsarme Lebensweise des Menschen führt zu einem Wegfall von Stimuli («Stressoren»), die für die Homöostase notwendig sind. Die Therapiemittel der Physikalischen Therapie bieten die Möglichkeit, fehlende Stimuli zu ersetzen. Unter einer solchen Behandlung bleiben die komplexen Regelkreise vollständig erhalten. Bei einer medikamentösen Therapie besteht immer das Risiko einer Übersteuerung und von starken Gegenregulationen, die sich als unerwünschte Wirkungen präsentieren.

Der Mensch ist mit einer grossen Zahl von Mechanismen ausgestattet, die ihm erlauben, sich an verschiedenste Situationen rasch und effizient anzupassen. Die Mechanismen widerspiegeln die Evolution. Eine ungeeignete Lebensweise kann mitunter sehr schwerwiegende Folgen haben. Umgekehrt können schon kleine Änderungen der Lebensweise, z.B. ein regelmässiges Training mit anschliessendem Saunabesuch, erstaunliche Wirkungen haben. Nicht zu vergessen: Der Mensch ist ein geselliges Wesen. Gesellschaft gehört zu seiner Natur. Ein Mangel an Kontakten kann ebenfalls depressiv machen (unter anderem auch eine Erfahrung des Schreibenden).

\section{Literatur}

1 Craft L, et al: The benefits of exercise for the clinically depressed. Prime Care Companion J Clin Psychiatry 2004;6:104-111.

2 Shevchuk NA: Adapted cold shower as a potential treatment for depression. Med Hypotheses 2008;70:995-1001.

3 Dinas PC, et al: Effects of exercise and physical activity on depression. Ir J Med Sci 2011; 180:319-325.
4 Szabo A, et al: Phenylethylamine, a possible link to the antidepressant effects of exercise. Br J Sports Med 2001;35:342-343.

5 Shevchuk NA: Possible use of repeated cold stress for reducing fatigue in chronic fatigue syndrome: a hypothesis. Behav Brain Funct 2007;3:55-60.

6 Tantimonaco M, et al: Physical activity and the endocannabinoid system: an overview. Cell Mol Life Sci 2014;71:2681-2698.
7 Andrews PW, et al: Is serotonin an upper or a downer? The evolution of the serotonergic system and its role in depression and the antidepressive response. Neurosci Biobehav Rev 2015;51:164-188. 